\title{
Adult familial nephronophthisis-spastic quadriparesia syndrome
}

INSERM

\section{Source}

INSERM. (1999). Orphanet: an online rare disease and orphan drug data base. Adult familial nephronophthisis-spastic quadriparesia syndrome. ORPHA:2666

This syndrome, associating familial adult medullary cystic disease with spastic quadriparesis has been described in two cases so far. Renal transplantation was successful in those two patients. 